\title{
Surgical debriefing: a reliable roadmap to completing the patient safety cycle
}

\author{
Scott L. Zuckerman, M.D., ${ }^{1}$ Daniel J. France, Ph.D., M.P.H., ${ }^{2}$ Cain Green, B.S., ${ }^{3}$ \\ Susie Leming-Lee, D.N.P., M.S.N., R.N., ${ }^{4}$ Shilo Anders, Ph.D., ${ }^{5}$ And J Mocco, M.D., M.S. ${ }^{1}$ \\ Departments of ${ }^{1}$ Neurological Surgery, ${ }^{2}$ Anesthesiology, and ${ }^{4}$ Perioperative Services, and ${ }^{5}$ Center for Research and \\ Innovation in Systems Safety, Vanderbilt University School of Medicine, Nashville; and ${ }^{3}$ College of Medicine, \\ The University of Tennessee Health Science Center, Memphis, Tennessee
}

\begin{abstract}
Morbidity and mortality due to preventable medical errors are a disastrous reality in medicine. Debriefing, a process that allows individuals to discuss team performance in a constructive, supportive environment, has been linked to improved performance in various medical and surgical fields, including improvements in specific procedures, teamwork and communication, and error identification. However, the neurosurgical literature on this topic is limited. The authors review the debriefing literature in the field of medicine, with a specific emphasis on the operating room, and they report their own institutional experience with a debriefing module, from invention to pilot implementation, at Vanderbilt University Medical Center. The authors share the challenges and lessons learned from their quality improvement project. The field of neurosurgery would undoubtedly benefit from embracing debriefing, as its potential has been established in other medical specialties and can serve as a valuable role in immediately learning from mistakes. The authors hope that their colleagues can learn from this experience and improve their own. (http://thejns.org/doi/abs/10.3171/2012.8.FOCUS12248)
\end{abstract}

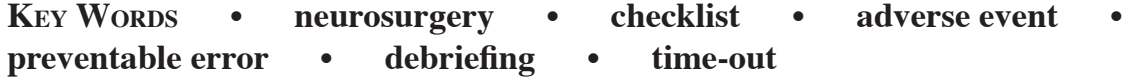

$\mathrm{O}$ F the more than 234 million surgical procedures performed each year, major complications occur between $3 \%$ and $17 \%$ of the time. ${ }^{15,19,42}$ Half of all surgical complications are thought to be avoidable, and most are the result of communication failures. Previous studies suggested that standardized checklists can facilitate perioperative team communication and eliminate potentially preventable errors. ${ }^{41}$

The checklists most frequently proposed as a way to help minimize patient safety risks in the perioperative environment are the preoperative time-out and the postoperative debriefing. The preoperative time-out was mandated by The Joint Commission and has been extensively studied. ${ }^{4,27}$ As part of The Joint Commission's initiative, hospital ORs conduct a time-out immediately before the planned procedure is initiated, where essential identifying patient and operative information is confirmed (http:// www.jointcommission.org/standards_information/npsgs. aspx). It also ensures that certain preoperative procedures are performed and that all questions or concerns are resolved..$^{13,14}$ As a result of numerous patient safety efforts, the time-out is currently standard of care.

Conversely, postoperative debriefing, another patient

\footnotetext{
Abbreviations used in this paper: $\mathrm{CRM}=$ crew resource management; $\mathrm{OR}=$ operating room; $\mathrm{PACU}=$ postanesthesia care unit; SBAR = Situation-Background-Assessment-Recommendation; VUMC $=$ Vanderbilt University Medical Center.
}

safety checklist, is rarely studied in the perioperative patient safety literature. Debriefing is defined as a process that allows "individuals to discuss individual and teamlevel performance, identify errors made, and develop a plan to improve their next performance" following a procedure or event. ${ }^{35}$

How a procedure is finished is as important as how it is started. Moreover, a fundamental maxim of medicine is for physicians to learn from our mistakes. Debriefing allows for the identification of failures, near failures, and successes, and provides an experiential learning modality. ${ }^{26}$ This act of reflection has been shown to be a key element of adult learning, ${ }^{25}$ and it is for this purpose that debriefing plays a critical role in fighter pilot training. Similar to military combat, surgery is a high-stakes environment, where mistakes can have drastic consequences. The surgical team's goal is to minimize mistakes and repeat them with lessening frequency. Debriefing has been shown to aid in reducing communication difficulties, which have been deemed the primary cause of human error in the surgical setting. ${ }^{40}$ Debriefing has also been linked to a reduction in adverse events and enhanced technical function in a surgical setting. ${ }^{3}$ It is for these reasons that the role of debriefing after a medical procedure-and particularly surgery-has begun to receive attention in the medical literature. We review the literature on debriefing measures in the field of medicine, with a specific emphasis on the operating room. ${ }^{24}$ 


\section{S. L. Zuckerman et al.}

\section{Debriefing}

\section{The Process of Debriefing}

Debriefing has been an important performance improvement tool in the military since its introduction during World War II, when it was used to question soldiers returning from a mission. ${ }^{37}$ Briefing and debriefing were established practice among fighter pilots years before CRM was initiated by NASA (National Aeronautics and Space Administration) in $1979 .{ }^{25}$ Since this time, debriefing has been shown to be an effective educational tool not only in military training but also in medical training. ${ }^{1,2,6,33}$

There are several systems-based guidelines that define the debriefing process for health care. Specifically, Salas et al. ${ }^{35}$ provided 12 "best practices" to ensure that debriefs are as effective as possible in a variety of medical settings, including the OR. These best practices emphasize creating an environment that is supportive of both the individual and the team, providing objective, focused learning, giving different types of feedback (outcome, process, individual, and team oriented) soon after performance, and making a record of goals to aid in future debriefs. Similarly, Owen and Follows ${ }^{29}$ summarized the key elements of the debriefing process through the mnemonic "GREAT," which stands for Guidelines, Recommendations, Events, Analysis, and Transfer (of knowledge to clinical practice).

Debriefing can also be done on an individual basis or within the context of a team. Boet et al. ${ }^{9}$ compared selfdebriefing with instructor debriefing by randomizing participants to either group. The authors found no significant difference between groups, emphasizing that there are many ways to conduct a successful debrief that maximizes learning. Furthermore, video recordings can catalyze the debriefing process. Hamilton et al. ${ }^{17}$ used video recordings to improve team function in simulated trauma resuscitations and achieved positive results: $90 \%$ of PGY-2 (2nd postgraduate year) general surgery residents thought that the exercise helped improve their teamwork and clinical proficiency. After simulated trauma resuscitations, a debriefing session was conducted 1 week later, which consisted of video feedback and didactic clinical instruction in deficient areas of knowledge. The next week, residents participated in a second video-recorded simulation, and all scores improved, with the lowest scoring area improving the most. The authors concluded that debriefing with a team leader served a vital role in improving the resident knowledge base and team function.

However, in a real practice setting, the importance of debriefing can be forgotten. The way in which debriefing is perceived by trainees and trainers can differ drastically. Ahmed et al. ${ }^{3}$ interviewed 33 surgical personnel (surgeons, anesthesiologists, and OR nurses) on the nature of debriefing in the ORs of hospitals on 3 continents (in the US, United Kingdom, and Australia). All anesthesiologists responded that they routinely provided some kind of debriefing to their trainees. However, this was not the case for surgeons. The majority of trainees preferred debriefing either after a case or at the end of the day (as opposed to debriefing during a case) and preferred specific, nonjudgmental, objective feedback consisting of both positive and negative critiques. Many trainers, however, thought that feedback was best given during a procedure. To have sufficient time to debrief, the authors suggested instituting a "time-out for debriefing" similar to the "time-out" used for the preoperative briefing to allow trainees and trainers adequate time to learn from the procedure. As seen, Ahmed et al. showed the disconnect between how trainees valued debriefing compared with the trainer's perception of its utility.

\section{Debriefing in the Surgical Setting}

Debriefing in the OR represents a unique challenge. Compared with other medical environments, ${ }^{12}$ operative teams must be efficient to minimize OR costs and minimize the amount of time the patient is under anesthesia. Moreover, open communication can prove to be difficult when all members of the surgical team are wearing hats and masks. Several studies link debriefing to improved team communication ${ }^{7,40}$ and error identification, $, 5,24,30$ which are linked to decreased rates of morbidity and mortality.

With multiple parties in the OR at all times, it is important to note how each party contributes or detracts from a successful debriefing. Robinson et al. ${ }^{34}$ studied which factors during the preparatory phase of implementing operating room debriefing plan were linked to program success at 102 facilities. The authors found that participation of OR nurse managers during the preparatory phase had a greater correlation with program success than participation of either hospital leadership or physicians. In 2009 Paull et al. ${ }^{31}$ collected data from 64 Veterans Health Administration facilities and found that the involvement of physicians and hospital leadership and the implementation committee correlated with more widespread briefing/debriefing implementation. A second article by Paull et al. ${ }^{32}$ showed increased compliance with both antibiotics and deep venous thrombosis prophylaxis after implementation of the checklist with the aforementioned 3 parties involved.

Two key components to debriefing compliance and efficacy are successful timing - when to hold the debriefing sessions-and implementation. Makary et al. ${ }^{24}$ reported debriefing immediately after a procedure at The Johns Hopkins Hospital ORs, ICUs, and other clinical environments using a brief postoperative checklist. The rationale for debriefing immediately was that the information was still close at hand. This debriefing regimen resulted in identifying various equipment and instrumentation dangers and developing measures to prevent future occurrences, such as adding a foot board to OR tables to prevent obese patients from falling off the table during gastric bypass surgery.

Numerous studies have found a positive impact of the debriefing on teamwork and communication. Berenholtz et al. ${ }^{7}$ studied the implementation and use of briefing and debriefing tools in 37,113 cases in a large medical center OR. These tools took an average of 2.9 and 2.5 minutes to complete, respectively. After first counts were conducted, the circulating nurse initiated the debriefing tool while the surgeon was still present and reviewed problems encountered during the procedure related to quality, safety, 
and outcomes. Surveys showed that caregivers thought that debriefing enhanced communication (69\%) and improved teamwork (72\%). A noted barrier to overcome in implementation of this protocol was gaining physician and staff support.

Similarly, Wolf et al. ${ }^{40}$ reported the implementation of OR briefing and debriefing on general surgery and surgical specialties services as part of the medical team training program. All OR staff and physicians attended a 1-day training session prior to implementation of the new briefing/debriefing protocol, which consisted of sections for briefing, debriefing, case scoring, and follow-up plans for issues. Debriefings consisted of reviewing team performance and were performed after the operation while all team members were still present in the OR. A Safety Attitudes Questionnaire taken by staff members before and approximately 1 year after implementation of the briefing/debriefing protocol resulted in significant increases in working conditions and perceptions of management, as well as feelings of collegiality and improved patient safety. The briefing/debriefing protocol also reduced the number of cases involving delays or requiring follow-up and was linked to an increase in the case score. This study's success was partially attributed to frequent meetings between representatives from each discipline that resulted in prompt action regarding issues raised during debriefings.

Two studies showed improvements in safety-notably the identification of hazards in the OR-related to the use of OR debriefing. Papaspyros et al. ${ }^{30}$ showed that formal checklist-guided OR briefing and debriefing had positive effects on patient safety and teamwork in cardiac ORs in the United Kingdom. After the surgery, debriefing was performed, which could be as minimal as a compliment to a team member on a job well done. This debriefing process identified multiple recurring errors, such as faulty instruments, background chatter, and excessive OR traffic. Additionally, a survey of staff members yielded positive reactions from all, including assertions that the protocol improved communication and professionalism. Another study linking debriefings to discovery of safety hazards was published by Bandari et al. ${ }^{5}$ earlier this year. A briefing/debriefing tool was implemented in the ORs and identified 6202 defects (issues with instrumentation, radiology, laboratory, supply, and communication/safety, as well as other areas) during its 44 months of implementation, with $46 \%$ of the defects being identified during briefing and $54 \%$ during debriefing. The list of errors identified during briefing or debriefing was sent to administrative personnel for the specific surgical service weekly meeting and was provided on a monthly basis to administration. Staff members and physicians were informed of the steps being taken to resolve issues on a daily basis by the clinical outcomes nurse (for staff members) or the administrative nurse manger (physicians). This study showed that briefings and debriefings were a practical and successful means of identifying both clinical and operational errors in surgical care.

The value of debriefing cannot be underestimated. It has been linked to improved performance in various medical and surgical fields, including improvements in specific procedures, ${ }^{11,12}$ teamwork and communication, ${ }^{7,26,40}$ and error identification. ${ }^{5,30}$ For debriefing to be as effective as possible for all members involved, it should be conducted in a consistent manner, soon after a procedure and with all team members involved. Any issues identified during debriefing should be communicated to the proper hospital administration, which should ideally keep the OR staff updated on the progress of remedying the issue. Debriefing can be a powerful tool in creating team unity and awareness, as well as reducing errors, which in turn lead to a more enjoyable working environment for medical personnel and a safer operative experience for the patient.

\section{Debriefing: The Vanderbilt Experience}

\section{Background}

Nearly a decade ago, the administrative and clinical leadership at VUMC (located in Nashville, Tennessee) committed to improving teamwork and team communication in safety-critical clinical processes to meet their strategic goal of moving from a reactive safety culture to creating a preventative and just safety culture. This work, which is now engrained in the mission of the hospital, was originally motivated by recommendations put forth by the Institute of Medicine to adopt and translate safety practices from other high-risk domains, such as aviation and nuclear power, to improve patient safety. ${ }^{18,20}$ To jumpstart the initiative, hospital leadership procured the services of a commercial vendor in 2003 to train all clinicians at the Vanderbilt University Hospital and the Monroe Carell Jr. Children's Hospital in aviation CRM. Crew resource management training has been shown to improve team communication and decision making, facilitate team building, improve staff morale, and align work processes in aviation. ${ }^{36}$ The CRM training course was conducted over an 8-hour session and included lectures, case studies, and role playing. After the CRM training phase was completed, VUMC's Perioperative Services assumed leadership and the internal management of CRM tool development and process reengineering. Transferring the management and development of the program to the hospital was determined to be critical in securing long-term physician buy-in and engagement during the design and implementation phases. Descriptions and results of the commercial CRM training program and the subsequent internalization and evolution of the program in Perioperative Services have been published previously. ${ }^{13,14,16,21,23}$

The transformation of VUMC's safety culture and work processes to support and sustain patient-centered communications has been gradual and, at times, very difficult. It is also far from complete. However, the work has fostered continual organizational learning and has produced functional processes and structured tools for team communications in dynamic, high-risk clinical settings (Fig. 1). Examples include the development and implementation of 1) a structured preincision time-out using an electronic whiteboard to guide the time-out process in the $\mathrm{OR}^{23}$ and 2) a modified SBAR tool to improve the quality (both content and tone) of postsurgical patient handovers between anesthesia providers and nurses in the 
PACU. ${ }^{39}$ Recently, Mainthia et al. ${ }^{23}$ showed that surgical team compliance with the preprocedural time-out has increased from $47 \%$ to nearly $90 \%$ as a result of institution commitment.

\section{Development of Postoperative Debriefing}

A postoperative debriefing session has been a core component of VUMC's CRM toolkit since its inception in 2003 (Table 1). However, by 2010 certain patient safety events in the perioperative environment indicated that the debriefing process should be standardized to prevent process drift. An observational study of cardiac and neurosurgical cases determined that surgeons performed postsurgical debriefing in less than a quarter of surgical cases..$^{13}$ It was determined that the low compliance scores resulted from confusion regarding timing and ownership of the debriefing. While the expectation was that the surgeon would perform the debriefing prior to closing, participants reported that debriefings, when completed, were frequently performed by the charge nurse or another member of the team just prior to the patient's departure from the OR. It also became evident that the debriefing was perceived to delay closing and to interfere with standard surgeon workflow. Therefore, opportunities to correctly perform the debriefing were lost when the surgeon left the OR. To refocus this effort, the leadership of Perioperative Services in collaboration with the Perioperative
Quality Improvement Committee agreed to pilot the implementation of a revised debrief that would "hardwire" the patient safety checklist process, preventing process drift, while increasing the efficiency and effectiveness of documenting critical safety processes endorsed as 2010 National Safety Goals by The Joint Commission on Accreditation of Healthcare Organizations.

In November 2010, a multidisciplinary Perioperative Debrief Task Force, which consisted of surgeons, anesthesiologists, human factors experts, nurses, and OR staff, was deployed to create a succinct yet comprehensive surgical checklist to guide structured postoperative debriefings. A major design criterion was that the checklist tool must be able to support debriefings performed in ORs and PACUs. The National Safety Goals and Toyota Production System Lean $5 \mathrm{~S}$ principles (sorting, stabilizing, sweeping or shining, standardizing, and sustaining practice) and rules of use (activities, connections, pathways, and improvement) were used to form a framework to guide the design of a debrief checklist (http://www.jointcommis sion.org/standards_information/npsgs.aspx). ${ }^{38}$ Alignment with National Safety Goals was selected to improve the effectiveness of communication among caregivers and to improve the effectiveness of clinical alarm systems (that is, to enhance situational awareness) (http://www.joint commission.org/standards_information/npsgs.aspx). The Lean principles prescribe fixing the problem at the site

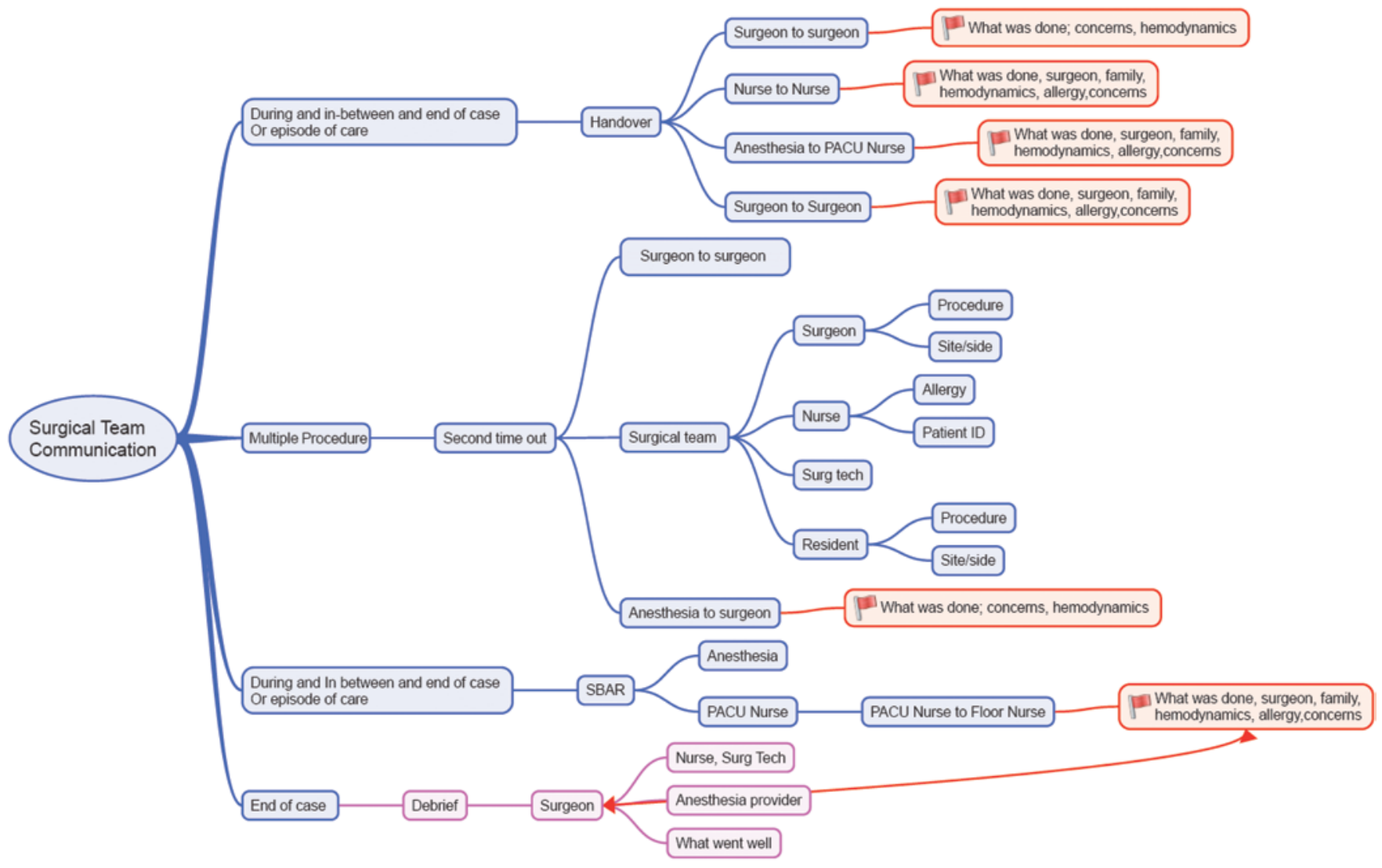

FIG. 1. Overview of surgical team communication processes, designed by Stephanie Randa and Susie Leming-Lee. ID = identification; Surg Tech = surgical technologist. "Family" refers to providing a general update to the family regarding the patient's status. The red flags indicate specific questions that should not be missed. 
Debriefing in neurosurgery

TABLE 1: Original CRM postoperative debriefing*

\begin{tabular}{|c|c|c|c|c|c|}
\hline \multicolumn{2}{|c|}{$\begin{array}{l}\text { Please Fill-in Participant's Name/s } \\
\text { Circulator: }\end{array}$} & \multicolumn{3}{|l|}{ Surgeon:_ } & \multirow[b]{3}{*}{$\mathrm{N} / \mathrm{A}$} \\
\hline \multicolumn{2}{|l|}{ Anesthesiologist: } & \multicolumn{3}{|l|}{ All Staff:_ } & \\
\hline WHO & & WHAT & Yes & No & \\
\hline Circulator & \multicolumn{5}{|c|}{ States status of first count and if counts correct } \\
\hline Surgeon/Resident Surgeon & \multicolumn{5}{|c|}{ Verifies counts } \\
\hline Surgeon/Resident Surgeon & \multicolumn{5}{|c|}{ Debriefs; reviews final procedure } \\
\hline Surgeon/Resident Surgeon & \multicolumn{5}{|c|}{ Assessment of outcome } \\
\hline Anesthesiologist/CRNA & \multicolumn{5}{|c|}{ States patient's status } \\
\hline Anesthesiologist/CRNA & \multicolumn{5}{|c|}{ States disposition of patient } \\
\hline Surgeon/Resident Surgeon & \multicolumn{5}{|c|}{ Invites all team members to give performance feedback } \\
\hline Surgeon/Resident Surgeon & \multicolumn{5}{|c|}{ Summarizes all inputs } \\
\hline Surgeon/Resident Surgeon & \multicolumn{5}{|c|}{ Thanks team for assisting with case } \\
\hline
\end{tabular}

* CRNA = certified registered nurse anesthetist.

where it occurs such that interruptions in the debriefing process result in delaying or canceling the surgical case until each item or task on the checklist is completed. Lean principles strive to empower workers (that is, clinicians and staff) to raise the alarm when deviations from safe practice occur. $^{28,38}$

The VUMC process, which aligns with national recommendations, requires the surgeon to call and conduct the debriefing prior to closing the surgical site. Unlike in the preprocedural time-out, debriefing cues the surgeon to invite performance feedback from the staff involved in the surgical case. Performance feedback is a brief postcase huddle led by the surgeon that allows surgical team members to voice their assessments of the team's safety performance during the case. In consideration of the process flow associated with the debriefing, the Perioperative Debrief Task Force members designed a pilot paper-based Surgical Debrief Checklist that integrated input from the neurosurgical team (surgeons, nurses, anesthesiologist, and staff members), The Joint Commission regulatory requirements, and the Lean principles over 3 Plan, Do, Study, Act (PDSA) improvement cycles.

As shown in Table 2, the following process steps were included in the surgical debriefing: status of the surgical counts; verification of the counts; review of the final procedure and diagnosis; patient's status and disposition; an invitation by the surgeon for all team members to give performance feedback; a summary of all inputs; and a "thank you" to the team from the surgeon or proceduralist for assisting in the case.

\section{Pilot: Neurosurgery}

In late 2011 a neurosurgery team volunteered to pilot this paper version of the Surgical Debrief Checklist. The neurosurgery team received "just in time training" to ensure all team members understood the purpose of the tool, the sequencing of the debriefing process steps, and the participants' roles. The Surgical Debrief Checklist was enlarged and posted in 4 neurosurgery ORs during the training period and prior to the pilot study. At the end of a 6-week pilot the neurosurgery team reconvened to provide feedback on the positives and what needed improvement on the debrief checklist. The team, which did not include the surgeons, indicated the need to streamline the checklist. The revised streamlined tool, which is shown in Table 3, is scheduled to be tested in the fall of 2012.

As the Surgical Debrief Checklist continues to evolve, the Department of Anesthesiology's Perioperative Computing is working in parallel with the Center for Research and Innovation in Systems Safety to adapt the paperbased checklist into an electronic checklist, which will be integrated within the Vanderbilt Perioperative Information System and displayed on electronic whiteboards positioned in each OR. This final stage of development and testing will include extensive human factors analyses and usability testing to ensure the tool is intuitive, easy to use, and embedded into surgical workflow processes.

\section{Conclusions}

The value of debriefing has been strongly established. It has been linked to improved performance in various medical and surgical fields, including improvements in specific procedures, ${ }^{11,12}$ teamwork and communication, $7,26,40$ and error identification. ${ }^{5,32}$ In order for debriefing to be as effective as possible, it should be conducted in a consistent manner, soon after a procedure, and with all team members involved. Debriefing can be a powerful tool in creating team unity and awareness, as well as reducing errors, which in turn leads to a more enjoyable working environment for medical personnel and a safer operative experience for the patient.

However, the successful design of a debriefing module is not tantamount to successful implementation, and the implementation of a debriefing module has proved challenging. 6,10,11,24 Poor compliance is most likely multifactorial, resulting from a medical culture prided on individuality and expertise, insufficient research on where 


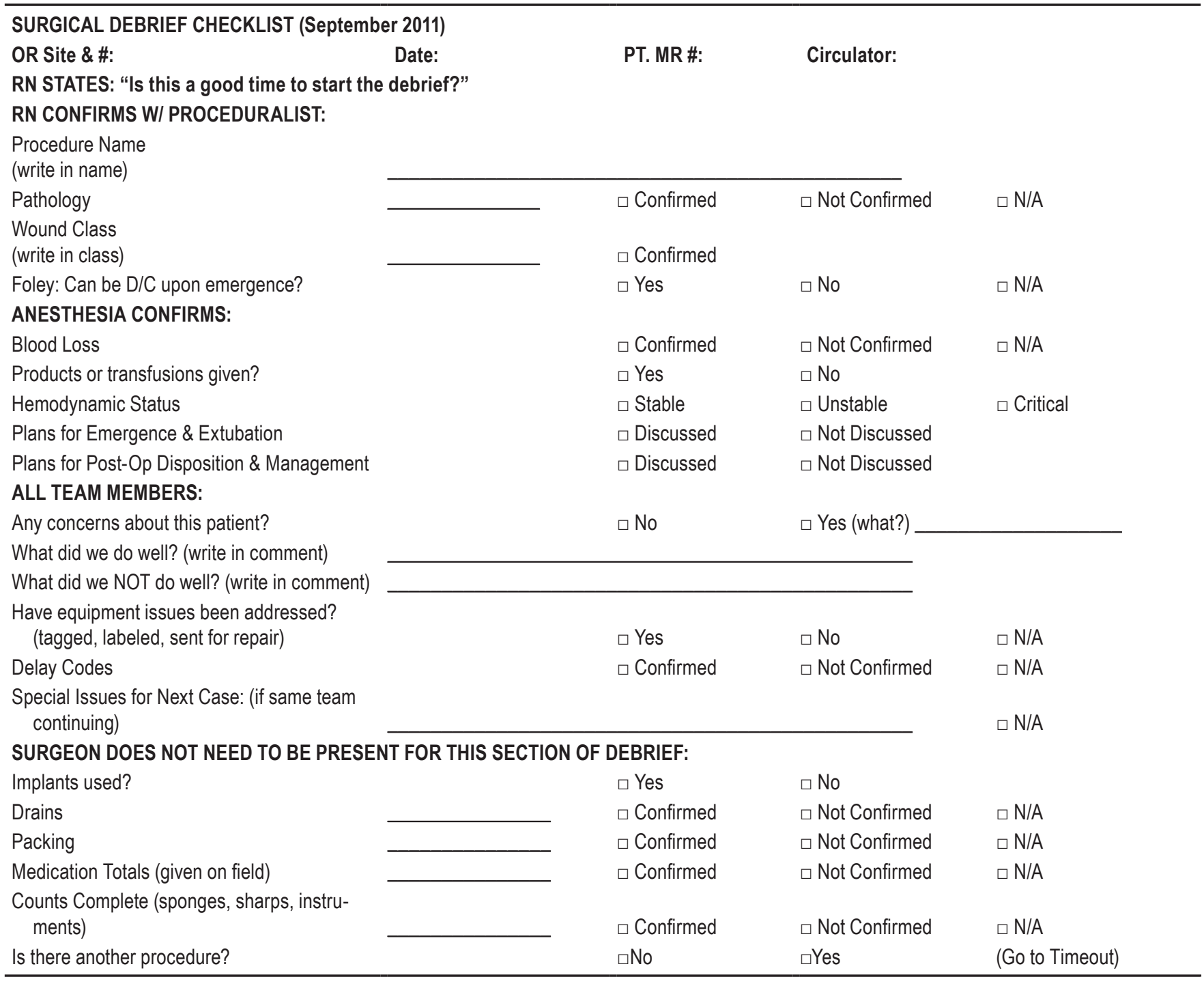

* $\mathrm{D} / \mathrm{C}=$ discontinued; PT. MR \# = patient medical record number; $\mathrm{N} / \mathrm{A}$ = not applicable; $\mathrm{RN}$ = registered nurse.

and when to use checklists, and insufficient knowledge on their value in improving patient outcomes. ${ }^{8}$ Levy et al. ${ }^{22}$ showed that despite $100 \%$ recorded compliance with a preincision checklist in 142 pediatric surgical cases, none completely executed all items on the checklist. When direct observation is used, Zuckerman et al. ${ }^{43}$ showed that 2 separate observer groups of nurses and medical students recorded different compliance rates when evaluating the preoperative time-out.

We have learned from our institutional experience and have identified several potential barriers to a debriefing application. One is surgeon buy-in. More applicable in older generations, the OR is a place of routine, and any institution-mandated change in a surgeon's practice is often met with resistance. This resistance often carries over to the design process. Anecdotally, we have found that surgeon buy-in at the program's inception greatly improves surgeon and department enthusiasm in implementing quality practice. Chairman buy-in has also helped compliance. Second, attending surgeons will often exit a case early (prior to skin closure), especially in academic medical centers, leaving the question of when to conduct a debriefing. Realizing the reality of academic practice, it behooves any debriefing program to recognize an attending's early exit, not fight it, and integrate it into a debriefing program. Third, administrative infrastructure is needed to use information gleaned from debriefing to change practice. Lastly, electronic integration has been shown to increase compliance. As mentioned, transitioning the presurgical time-out from a nurse's computer to an electronic whiteboard increased compliance from $47 \%$ to $90 \%$ at our institution. ${ }^{23}$ Applying the debriefing process to an electronic whiteboard for all OR members to see has potential to drastically improve practice and is the next step in our ongoing quality projects.

Although the surgical debriefing literature has yet to definitively link debriefing with reduced rates of morbidity and mortality, it has shown decreased error rates and 
Debriefing in neurosurgery

TABLE 3: Current streamlined Surgical Debrief Checklist

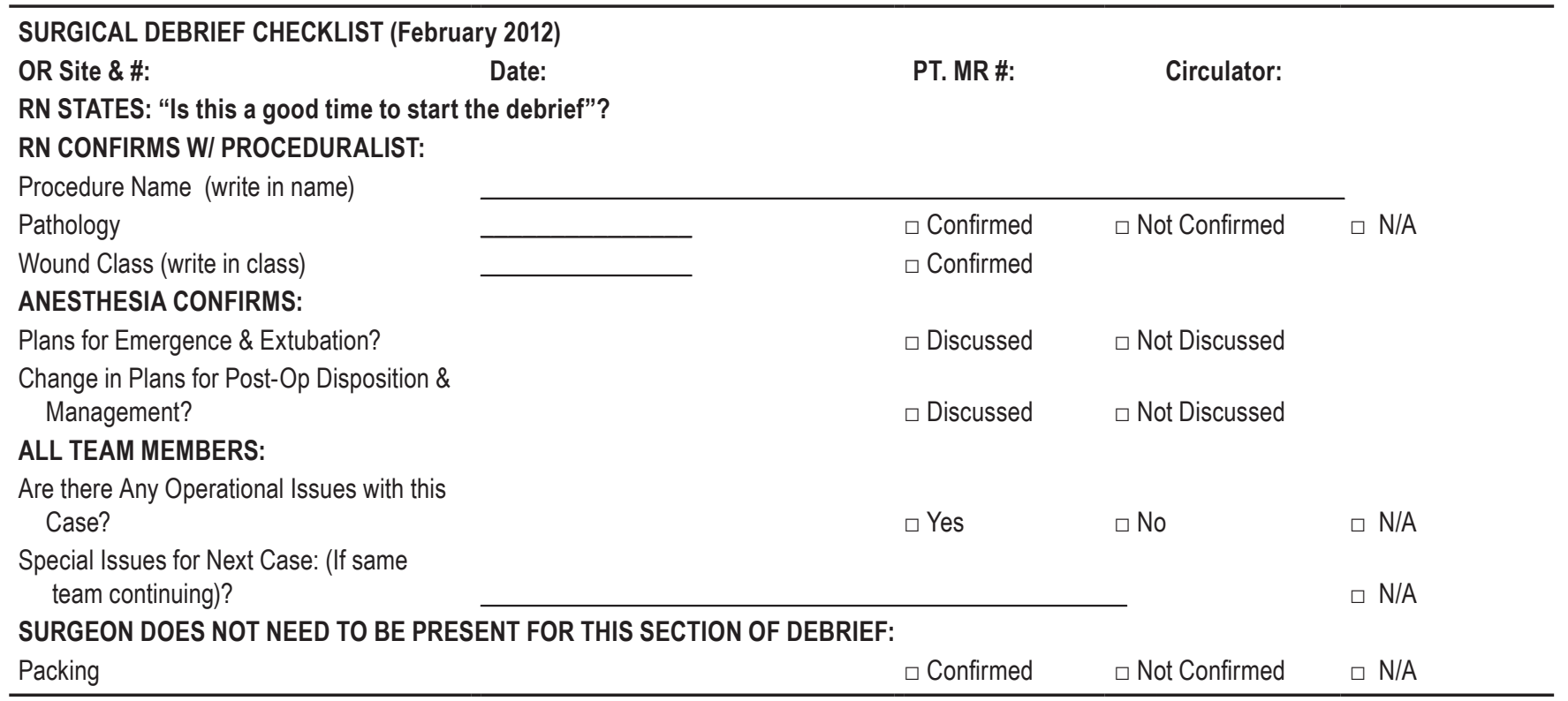

improvements in team communication. The neurosurgical literature on this topic, however, is even more limited. We report our own experience and challenges with a debriefing module, from invention to pilot implementation. Debriefing has potential to serve a valuable role in the culture of safe surgery. We hope our colleagues can learn from our experience and improve their own.

\section{Disclosure}

Dr. Mocco is a consultant for Lazarus Effect, NFocus, and Edge Therapeutics. He owns stock in Blockade Therapeutics. The authors report no conflict of interest concerning the materials or methods used in this study or the findings specified in this paper.

Author contributions to the study and manuscript preparation include the following. Conception and design: Mocco, Zuckerman, France, Green, Leming-Lee. Analysis and interpretation of data: Leming-Lee, Anders. Drafting the article: Zuckerman, France, Green, Leming-Lee, Anders. Critically revising the article: Mocco, Zuckerman, France. Reviewed submitted version of manuscript: Mocco, Zuckerman, France, Green. Administrative/technical/material support: Zuckerman, Green, Leming-Lee, Anders. Study supervision: Mocco, Zuckerman.

\section{Acknowledgment}

The authors acknowledge Stephanie Randa for the development of Fig. 1.

\section{References}

1. Adler AB, Bliese PD, McGurk D, Hoge CW, Castro CA: Battlemind debriefing and battlemind training as early interventions with soldiers returning from Iraq: randomization by platoon. J Consult Clin Psychol 77:928-940, 2009

2. Adler AB, Castro CA, McGurk D: Time-driven battlemind psychological debriefing: a group-level early intervention in combat. Mil Med 174:21-28, 2009

3. Ahmed M, Sevdalis N, Paige J, Paragi-Gururaja R, Nestel D, Arora S: Identifying best practice guidelines for debriefing in surgery: a tri-continental study. Am J Surg 203:523-529, 2012

4. American College of Surgeons: Wrong site surgery and the Universal Protocol. Bull Am Coll Surg 91:63, 2006
5. Bandari J, Schumacher K, Simon M, Cameron D, Goeschel CA, Holzmueller CG, et al: Surfacing safety hazards using standardized operating room briefings and debriefings at a large regional medical center. Jt Comm J Qual Patient Saf 38: 154-160, 2012

6. Barker J: Error reduction through team leadership: what surgeons can learn from the airline industry. Clin Neurosurg 54:195-199, 2007

7. Berenholtz SM, Schumacher K, Hayanga AJ, Simon M, Goeschel C, Pronovost PJ, et al: Implementing standardized operating room briefings and debriefings at a large regional medical center. Jt Comm J Qual Patient Saf 35:391-397, 2009

8. Berger DH: Crisis checklists for the operating room: development and pilot testing. J Am Coll Surg 213:218-219, 2011

9. Boet S, Bould MD, Bruppacher HR, Desjardins F, Chandra $\mathrm{DB}$, Naik VN: Looking in the mirror: self-debriefing versus instructor debriefing for simulated crises. Crit Care Med 39:1377-1381, 2011

10. Bosk CL, Dixon-Woods M, Goeschel CA, Pronovost PJ: Reality check for checklists. Lancet 374:444-445, 2009

11. Corbett N, Hurko P, Vallee JT: Debriefing as a strategic tool for performance improvement. J Obstet Gynecol Neonatal Nurs 41:572-579, 2012

12. Edelson DP, Litzinger B, Arora V, Walsh D, Kim S, Lauderdale DS, et al: Improving in-hospital cardiac arrest process and outcomes with performance debriefing. Arch Intern Med 168:1063-1069, 2008

13. France DJ, Leming-Lee S, Jackson T, Feistritzer NR, Higgins MS: An observational analysis of surgical team compliance with perioperative safety practices after crew resource management training. Am J Surg 195:546-553, 2008

14. France DJ, Stiles R, Gaffney EA, Seddon MR, Grogan EL, Nixon WR Jr, et al: Crew resource management training-clinicians' reactions and attitudes. AORN J 82:213-224,2005

15. Gawande AA, Thomas EJ, Zinner MJ, Brennan TA: The incidence and nature of surgical adverse events in Colorado and Utah in 1992. Surgery 126:66-75, 1999

16. Grogan EL, Stiles RA, France DJ, Speroff T, Morris JA Jr, Nixon B, et al: The impact of aviation-based teamwork training on the attitudes of health-care professionals. J Am Coll Surg 199:843-848, 2004

17. Hamilton NA, Kieninger AN, Woodhouse J, Freeman BD, Mur- 


\section{S. L. Zuckerman et al.}

ray D, Klingensmith ME: Video review using a reliable evaluation metric improves team function in high-fidelity simulated trauma resuscitation. J Surg Educ 69:428-431, 2012

18. Institute of Medicine, Committee on Quality of Health Care in America: Crossing the Quality Chasm: A New Health System for the 21st Century. Washington, DC: National Academies Press, 2001

19. Kable AK, Gibberd RW, Spigelman AD: Adverse events in surgical patients in Australia. Int J Qual Health Care 14:269276, 2002

20. Kohn LT, Corrigan JM, Donaldson MS (eds): To Err Is Human: Building a Safer Health System. Washington, DC: National Academies Press, 2000

21. Leming-Lee S, France DJ, Feistritzer NR, Kuntz A, Sanders F, Higgins MS: Crew resource management in perioperative services: navigating the implementation road map. JCOM12:353-358, 2005

22. Levy SM, Senter CE, Hawkins RB, Zhao JY, Doody K, Kao LS, et al: Implementing a surgical checklist: more than checking a box. Surgery 152:331-336, 2012

23. Mainthia R, Lockney T, Zotov A, France DJ, Bennett M, St Jacques PJ, et al: Novel use of electronic whiteboard in the operating room increases surgical team compliance with preincision safety practices. Surgery 151:660-666, 2012

24. Makary MA, Holzmueller CG, Sexton JB, Thompson DA, Martinez EA, Freischlag JA, et al: Operating room debriefings. Jt Comm J Qual Patient Saf 32:407-410, 2006

25. McGreevy JM, Otten TD: Briefing and debriefing in the operating room using fighter pilot crew resource management. J Am Coll Surg 205:169-176, 2007

26. Miller KK, Riley W, Davis S, Hansen HE: In situ simulation: a method of experiential learning to promote safety and team behavior. J Perinat Neonatal Nurs 22:105-113, 2008

27. Norton E: Implementing the universal protocol hospital-wide. AORN J 85:1187-1197, 2007

28. Ohno T: Toyota Production System: Beyond Large-Scale Production. Portland, OR: Productivity Press, 1988

29. Owen H, Follows V: GREAT simulation debriefing. Med Educ 40:488-489, 2006

30. Papaspyros SC, Javangula KC, Adluri RK, O’Regan DJ: Briefing and debriefing in the cardiac operating room. Analysis of impact on theatre team attitude and patient safety. Interact Cardiovasc Thorac Surg 10:43-47, 2010

31. Paull DE, Mazzia LM, Izu BS, Neily J, Mills PD, Bagian JP: Predictors of successful implementation of preoperative briefings and postoperative debriefings after medical team training. Am J Surg 198:675-678, 2009

32. Paull DE, Mazzia LM, Wood SD, Theis MS, Robinson LD, Carney B, et al: Briefing guide study: preoperative briefing and postoperative debriefing checklists in the Veterans Health Administration medical team training program. Am J Surg 200:620-623, 2010
33. Pischke PJ, Hallman CJ: Effectiveness of critical event debriefings during Operation Iraqi Freedom II. US Army Med Dep J Jul-Sep:18-23, 2008

34. Robinson LD, Paull DE, Mazzia LM, Falzetta L, Hay J, Neily J, et al: The role of the operating room nurse manager in the successful implementation of preoperative briefings and postoperative debriefings in the VHA Medical Team Training Program. J Perianesth Nurs 25:302-306, 2010

35. Salas E, Klein C, King H, Salisbury M, Augenstein JS, Birnbach DJ, et al: Debriefing medical teams: 12 evidence-based best practices and tips. Jt Comm J Qual Patient Saf 34:518527, 2008

36. Salas E, Wilson KA, Burke CS, Wightman DC: Does crew resource management training work? An update, an extension, and some critical needs. Hum Factors 48:392-412, 2006

37. Schenarts PJ: Debriefing is an effective method for providing feedback and ensuring adherence to best clinical practice by residents in the intensive care unit. Crit Care Med 35:957958, 2007

38. Spear S, Bowen HK: Decoding the DNA of the Toyota Production System. Harv Bus Rev 77:96-106, 1999

39. Weinger MB, Slagle J, Kuntz A, Banerjee A, Schildcrout J, Mercaldo N, et al: Improving actual handover behavior with a simulation-based training intervention. Presented at the 54th annual meeting of the Human Factors and Ergonomic Society (http://pro.sagepub.com/content/54/12/957) [Accessed September 28, 2012]

40. Wolf FA, Way LW, Stewart L: The efficacy of medical team training: improved team performance and decreased operating room delays: a detailed analysis of 4863 cases. Ann Surg 252:477-485, 2010

41. World Alliance for Patient Safety: WHO surgical safety checklist and implementation manual. (http://www.who int/patientsafety/safesurgery/tools_resources/SSSL_Manual_ finalJun08.pdf) [Accessed September 25, 2012]

42. World Health Organization: 10 facts on safe surgery. (http:// www.who.int/features/factfiles/safe_surgery/en/index.html) [Accessed September 25, 2012]

43. Zuckerman SL, Poon SA, Mainthia R, Hagan SL, Lockney TD, Zotov A, et al: Methodology and bias in assessing compliance with a surgical safety checklist. Jt Comm J Qual Patient Saf [in press], 2012

Manuscript submitted July 15, 2012.

Accepted August 28, 2012.

Please include this information when citing this paper: DOI: 10.3171/2012.8.FOCUS12248.

Address correspondence to: J Mocco, M.D., M.S., VUMC Department of Neurological Surgery, 1211 Medical Center Drive, Nashville, Tennessee 37232. email: j.mocco@vanderbilt.edu. 